Phyllis Dalley, Ph. D.

Faculté d'éducation, Université d'Ottawa

\title{
La formation à l'enseignement
en Ontario français à l'enseignement
en Ontario français
}

Mélissa Villella, M.A.

Faculté d'éducation, Université d'Ottawa

\section{Chronique internationale}

\section{Le système scolaire ontarien}

Pour des raisons sociohistoriques, l'éducation au Canada est une responsabilité provinciale et territoriale plutôt que nationale. Ainsi, depuis le début de la Confédération, et bien avant pour certaines provinces, l'élaboration et la mise en œuvre des systèmes scolaires (structures, politiques, programmations, normes de réussite, formation à l'enseignement, pour ne mentionner que ceux-là) suivent des logiques ayant des particularités provinciales et territoriales. Seul un système pancanadien, celui prévu pour les Premières Nations, les Métis et les Inuits, fait exception à cette décentralisation scolaire. Le présent article traite du système scolaire de la province de l'Ontario et, plus particulièrement, de la formation à l'enseignement offerte dans les facultés d'éducation de cette province.

Dans les prochaines sections, nous abordons, tour à tour, la structure du système scolaire ontarien, la structure du système de formation à l'enseignement et les défis et les possibilités d'avenir. Bien que nous traitions de l'ensemble du système, nous porterons une attention particulière à l'évolution de l'éducation en langue française, l'Ontario étant une province à majorité anglophone, et aux défis qui lui sont propres.

\section{Structure du système scolaire ontarien}

Aujourd'hui, le système scolaire ontarien est constitué d'un ministère de l'Éducation provincial, de conseils scolaires et 
d'écoles publiques financées à majorité par des fonds publics et d'autres écoles, par des fonds privés. Cet article n'abordera que le marché des écoles publiques de langue française.

On l'aura deviné, plusieurs choix s'offrent aux parents, tutrices et tuteurs en matière d'établissements scolaires publics pour leurs enfants. De prime abord, il existe des écoles primaires et secondaires de langue anglaise, non confessionnelles ou confessionnelles. Parmi ce nombre, on compte plusieurs écoles secondaires ayant une concentration particulière (sports, sciences, technologie, arts...). Les élèves de ces écoles suivent un nombre de cours plus élevé que la norme dans une concentration. Les écoles confessionnelles sont catholiques, sauf pour une seule école protestante. Certains parents, tutrices ou tuteurs ont également un droit constitutionnel d'inscrire leurs enfants dans une école confessionnelle (catholique) ou non confessionnelle de langue française. Là aussi, plusieurs écoles avec spécialisation ont vu le jour depuis les dix dernières années. Le graphique ci-dessous donne un aperçu global du système.

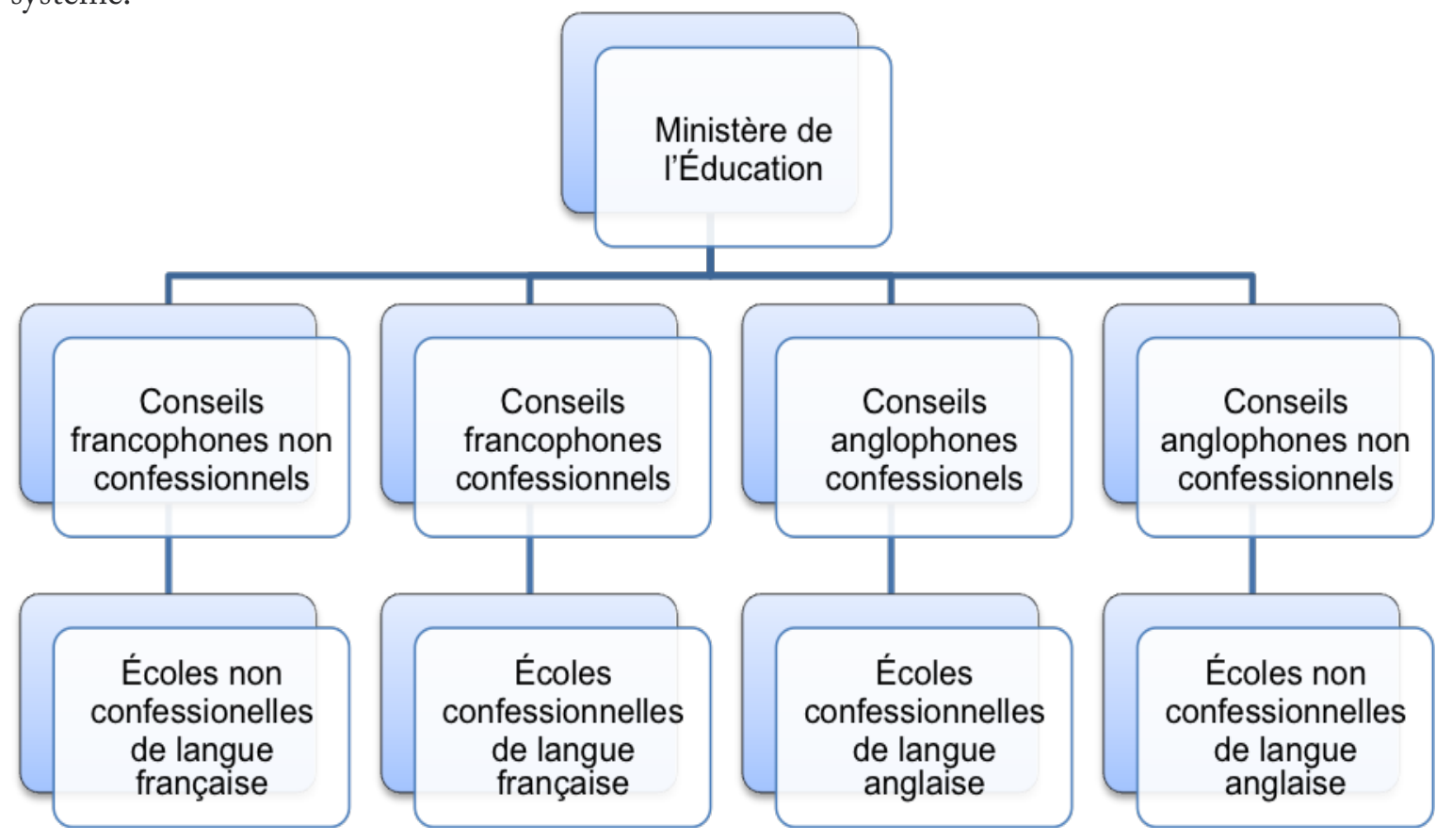

\section{La formation à l'enseignement}

En Ontario, 16 universités offrent un programme de formation à l'enseignement. De ces universités, trois offrent un programme en français à l'intention des futurs enseignantes et enseignants des écoles primaires et secondaires de langue française de la province. Des années 1970 à 2015, le cadre de formation à l'enseignement prévoyait l'obtention d'un premier diplôme universitaire suivi d'une année de formation professionnelle. À compter de la rentrée universitaire 2015, la formation professionnelle est passée à deux ans, toujours à la suite d'un premier diplôme, pour un total de cinq ou six années d'études universitaires. Ce changement permet de bonifier l'offre de cours, tant au niveau des didactiques que des fondements en éducation, d'une part, et d'augmenter le nombre de jours de stages pratiques dans les écoles, d'autre part. 
En Ontario, le baccalauréat en éducation s'organise autour des cycles scolaires que sont le primaire (la maternelle à la $3^{\mathrm{e}}$ année), le moyen (la $4^{\mathrm{e}}$ à la $6^{\mathrm{e}}$ année), l'intermédiaire (la $7^{\mathrm{e}}$ à la $9^{\mathrm{e}}$ année) et le supérieur (la $10^{\mathrm{e}}$ à la $12^{\mathrm{e}}$ année). Les étudiantes et les étudiants reçoivent une formation initiale dans deux cycles scolaires, soit le primaire-moyen, le moyen-intermédiaire ou l'intermédiaire-supérieur. Au cours de la première année de formation professionnelle, les étudiants suivront un total de 21 semaines de cours, 10 jours de stage d'initiation professionnelle ${ }^{1}$ et de 30 jours de stages pratiques d'enseignement. Pour ce qui est de la deuxième année, la durée des stages est de 50 jours et les cours de 15 semaines. Les cours dispensés donnent accès au savoir et à certains savoir-faire nécessaires à la pratique de l'enseignement alors que les stages pratiques sont nécessaires à la mise en pratique et à la bonification de ces savoirfaire dans deux cycles scolaires. Le stage d'initiation professionnelle a pour but principal de familiariser les étudiants avec la culture scolaire de l'Ontario français et avec les responsabilités professionnelles du personnel enseignant dans ce contexte. Intégrée à un cours en gestion de classe, cette initiation sera l'occasion de faire un aller-retour entre l'observation en milieu scolaire et la réflexion sur ses propres croyances et attentes vis-à-vis le rapport personnel enseignant/élève. Cette expérience répondra à une diversité de besoins, reflets de la diversité des parcours antérieurs des stagiaires. Nous y reviendrons dans la prochaine section portant sur les enjeux et les défis de la formation à l'enseignement en langue française.

\section{Enjeux et défis de la formation à l'enseignement en langue française}

Dans cette section, nous proposons de brosser le tableau de deux enjeux - la pénurie du personnel enseignant et l'inclusion réussie des étudiantes et étudiants d'origine immigrante à la profession enseignante - et de deux défis, la situation sociolinguistique de l'Ontario français et l'évaluation et la formation linguistiques du futur personnel enseignant. Loin d'être des enjeux et des défis isolés, ils s'imbriquent l'un dans l'autre.

\section{Une pénurie s'annonce}

Bien que la qualité de la formation à l'enseignement soit un facteur dans la décision de passer d'une formation professionnelle de un an à une formation de deux ans, l'état actuel du marché d'emploi a également joué dans la balance. En effet, du côté du système scolaire anglophone, le marché de travail est saturé et plusieurs nouveaux enseignantes et enseignants formés pour ce système y mettent 5 ans avant de trouver le plein emploi. Or, la situation n'est pas le même du côté francophone. En fait, devant la difficulté à pourvoir les postes vacants dans les écoles de langue française de certaines régions, plusieurs enseignent avec un permis spécial, c'est-à-dire sans avoir obtenu une formation reconnue à l'enseignement dans une école de langue française. Puisque la nouvelle formule diminue le nombre de nos finissants à l'Université d'Ottawa à 36 pour l'année universitaire 2015-2016 et de 150 à 200 par année subséquente, la pénurie ne pourra que s'aggraver. De plus, il existe une pénurie, parfois plus grande, d'enseignantes et d'enseignants du côté des écoles de langue française dans l'Ouest canadien, et nos finissantes et finissants sont sollicités de plus en plus de ce côté. Une formule pensée en fonction des besoins de la majorité risque donc d'avoir pour effet d'augmenter le nombre de permis locaux accordés

1 Pour des raisons institutionnelles, ce stage est intitulé « expérience d'engagement en milieu communautaire », bien qu'il ait lieu en milieu scolaire. 
pour l'enseignement dans les écoles de la minorité linguistique. Sans nier les grandes compétences de certains membres de cette catégorie d'enseignants, il est clair que sans avoir obtenu formation professionnelle adéquate, il est difficile de croire qu'il leur sera possible de contribuer efficacement à l'amélioration du rendement des élèves, toujours en deçà de la moyenne provinciale dans les évaluations nationales et internationales ni d'augmenter la confiance du public face à la qualité de l'éducation. En fait, plus qu'une question de professionnalisation, la pénurie en enseignement risque d'être un enjeu de société important pour la minorité francophone, une population déjà aux prises avec les effets de la minorisation.

\section{Un contexte sociolinguistique}

La minoration (ou minorisation) linguistique est [...] la réduction, selon des degrés divers, de l'exercice sociétal normal (et donc des domaines communicationnels) d'une langue. Une réduction pouvant remonter aux origines mêmes de son émergence mais le plus souvent fruit d'une domination subie par une communauté linguistique au cours d'une plus ou moins longue période et selon des modalités plus ou moins violentes. (Boyer, 2006, p. 261)

En Ontario, la minoration du français est le fruit de plusieurs années de politiques répressives, notamment le refus de l'accès à l'éducation en langue française, et de luttes politiques et juridiques pour la reconnaissance de cette langue en milieux scolaire, hospitalier et gouvernemental. Alors que le statut $\mathrm{du}$ français est aujourd'hui bien plus élevé que par le passé, il n'est pas pour autant langue légitime dans tous les domaines de la vie et les francophones ont toujours tendance à accepter la légitimité plus grande de la langue de la majorité en situation de contact linguistique bilingue ou plurilingue. Chez les élèves des écoles de langue française, cette minoration a un effet certain sur leur répertoire linguistique et leur propension à utiliser l'anglais en milieu scolaire et hors scolaire : dans plusieurs contextes, les élèves parlent plus souvent anglais ou franglais que français en salle de classe, dans les couloirs et dans la cour d'école. En fait, leur bilinguisme français-anglais donne souvent naissance à une pratique transcodique peu légitime en milieu unilingue : "Translanguaging is the process of making meaning, shaping experiences, gaining understanding and knowledge through the use of two languages' » (Baker, 2011, p. 288). Cette pratique «franglaise » est l'utilisation simultanée de l'anglais et du français dans une même situation de communication, que ce soit au niveau de la phrase, d'un passage ou encore de l'ensemble de la situation. Cette réalité sociolinguistique fait poindre des responsabilités en matière d'enseignement d'un français standardisé dans l'ensemble des matières scolaires et de respect de la variation linguistique du terrain.

L'Ontario français étant de plus en plus une communauté d'accueil de l'immigration francophone de par le monde, la Faculté d'éducation de l'Université d'Ottawa est devenue un lieu de rencontre de personnes parlant et valorisant des parlers français divers. Cette situation n'est pas sans causer des tensions entre groupes d'étudiantes et d'étudiants : nos salles de classe sont des lieux de rencontre de cultures, mais également de français standards pluriels - le français de référence n'est pas le même au Congo, au Québec, en Ontario ou en France - mettant en exergue toute la problématique du rapport à (aux) langue(s) française(s) en milieu minoritaire. Tout en négociant les tensions dans nos propres cours, il nous incombe donc de former le personnel enseignant des écoles de langue française en vue d'une prise en compte des besoins d'apprentissage et d'appartenance d'élèves ayant des parcours de vie et des rapports au français standard canadien d'une grande variabilité. 
La Faculté d'éducation de l'Université d'Ottawa a choisi le stage d'initiation professionnel comme moyen, toujours partiel, d'initier les futurs enseignantes et enseignants, d'origines diverses, à cette réalité du terrain. Cette initiative, qui ne donne lieu à aucune évaluation sommative de l'enseignement, a d'abord été expérimentée en raison des plus grandes difficultés d'intégration de la population estudiantine d'origine africaine en milieu de stage. Tout en reconnaissant que ces difficultés pourraient trouver leur origine dans le milieu universitaire ou le milieu scolaire plutôt que chez les étudiantes et étudiants, la Faculté d'éducation détient un plus grand pouvoir d'action auprès de ces dernières et derniers et a choisi d'investir là où les probabilités de succès sont les plus grandes. L'expérience fut à ce point positive que nous avons décidé d'en généraliser la pratique.

\section{De l'immigration à l'enseignement en Ontario français}

Près du deux tiers des étudiantes et étudiants de l'Université d'Ottawa viennent soit du Québec, où le français est langue majoritaire, soit d'un pays étranger où un français standard est garant de mobilité sociale. L'autre tiers a évolué en Ontario, là où le français et les francophones sont minorés. Le stage d'initiation permettra aux étudiantes et aux étudiants qui ont connu l'école de langue française à titre d'élève de débuter leur transition vers une prise en compte professionnelle de l'école de langue française et du travail scolaire en milieu minoritaire et à prendre conscience des effets de l'immigration sur la composition de la population étudiante des écoles et sur les pratiques pédagogiques à privilégier dans un contexte diversifié. Pour la majorité des Québécois et Québécoises, cette première expérience en milieu minoritaire francophone risque fort d'entrainer un certain choc en raison des effets de la minoration du français en Ontario : habitués à un contexte où parler français va de soi, ils se trouveront devant le défi des pratiques langagières bilingues ou plurilingues des élèves qui remettent en question leur norme de référence unilingue française.

Pour leur part, nombreux sont les étudiantes et étudiants d'origines immigrantes à avoir des diplômes non reconnus par l'État canadien et être ainsi dans l'obligation de parfaire une nouvelle formation dans l'espoir de trouver un emploi ayant un statut comparable à celui détenu dans leur pays d'origine. L'incompréhension de cette clientèle devant les pratiques langagières des élèves des écoles de langue française en Ontario est d'autant plus grande que le français est une langue que l'on maitrise par la voie scolaire dans leurs pays d'origine et que la valeur sociale du français standard est telle que toute divergence de cette norme est considérée comme inacceptable. De plus, ces stagiaires se trouvent devant une culture scolaire fort différente de celle qu'elles ont connue au cours de leur enfance, notamment en ce qui a trait à la place de l'enfant au centre des préoccupations de l'école et, de ce fait, au rapport entre le personnel enseignant et les élèves. En fait, pour plusieurs, le statut d'enseignant confère une autorité à laquelle les élèves doivent se soumettre et la rencontre d'un milieu où les élèves peuvent prendre la parole et discuter impunément avec le personnel enseignant est difficile. Le stage d'initiation professionnelle doit leur permettre de vivre un passage plus graduel d'une situation où le respect de l'enseignant est incontestable à une situation où le personnel enseignant doit respecter les élèves et gagner leur respect. Alors que Mujawamariya notait en 2002 que ce sont les finissantes et finissants d'origines immigrantes qui rencontrent la plus grande difficulté à trouver le plein emploi dans une école de langue française, nous espérons que le stage d'initiation professionnelle, situé en début de parcours de formation professionnelle, puisse aider nos étudiantes et étudiants à prendre conscience 
de l'écart qui sépare leurs attentes de la réalité ontarienne dans une situation peu menaçante, le stage n’étant pas évalué.

\section{Quelles compétences linguistiques évaluer et acquérir?}

Si le contexte sociolinguistique de l'Ontario français a des retombées sur les pratiques langagières des jeunes, il a également pour effet de leur rendre l'accès aux études supérieures plus difficile que pour la majorité anglophone. Qui plus est, ceux et celles qui réussissent leur entrée à l'université et obtiennent un premier diplôme dans un programme dispensé en langue française ne réussissent pas tous l'examen de compétences linguistiques de notre Faculté d'éducation. En fait, il est estimé que près de $80 \%$ des étudiantes et étudiants ne réussissent pas cet examen d'entrée et se voient dans l'obligation de suivre un cours d'appoint et de réussir un nouvel examen avant d'obtenir leur baccalauréat en éducation.

Plusieurs questions se posent quant aux compétences linguistiques de ces étudiants. De prime abord, nous détenons peu de données démographiques sur les personnes qui écrivent l'examen et conséquemment sur qui le réussit et qui ne le réussit pas. En deuxième lieu, n’ayant pas mené d'étude des types d'erreurs commises lors de l'écriture de l'examen, nous ne pouvons dégager un portrait précis des compétences des étudiants. Un tel portrait permettrait tant aux étudiants qu'aux professeurs de mieux cibler leurs efforts d'apprentissage et d'enseignement. Finalement, il est difficile d'affirmer que les compétences évaluées sont celles nécessaires à la pratique de l'enseignement. Afin de répondre à cette question, il nous serait nécessaire de faire une analyse des pratiques littératiées en milieu professionnel et de les arrimer à notre évaluation et à notre enseignement. Tout comme nous nous devons de tenir compte de la diversité de notre clientèle et du contexte des écoles dans notre offre de cours et de stages, la diversité des pratiques du français serait également une donnée incontournable dans notre réflexion sur la formation linguistique offerte par la Faculté. En fait, en raison de la complexité de notre situation sociopolitique, les auteurs de cet article proposent qu'un regard sociolinguistique plutôt qu'uniquement linguistique sur l'ensemble de cette question soit de mise à ce moment de notre histoire.

\section{Conclusion}

La Faculté d'éducation de l'Université d'Ottawa a mis deux ans à restructurer nos programmes de formations à l'enseignement pour les écoles de langue française sur deux ans. Dans le domaine de l'éducation, une telle restructuration implique la tenue de rencontres avec les autres facultés d'éducation de la province, le ministère de l'Éducation, les conseils scolaires ainsi que l'Ordre des enseignantes et des enseignants de l'Ontario. L'Ordre étant responsable de l'agrément des programmes de formation à l'enseignement, il a également été nécessaire de lui soumettre les descriptions des cours et stages proposés ainsi que les plans de cours lorsque ceux-ci étaient disponibles. La structure étant maintenant en place grâce au travail acharné du directeur des programmes, il nous est maintenant possible de penser l'avenir en fonction des enjeux et défis identifiés dans cet article.

Pour ce faire, il nous semblerait intéressant de profiter des expériences d'autres universités dans les domaines de l'évaluation et de la formation linguistique dans une perspective sociolinguistique de la diversité des pratiques du français, de l'inclusion des stagiaires d'âge et d'horizons divers dans les écoles partenaires et dans la formation à l'autoréflexivité culturelle et linguistique pour un meilleur 


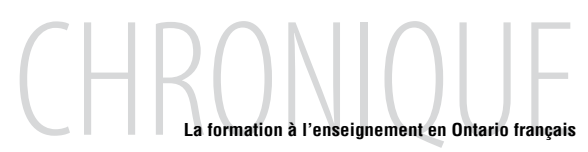

accueil pédagogique d'une francophonie plurielle. Que ce soit les étudiants issus du système scolaire ontarien ou d'un autre système scolaire, tous auront à enseigner dans un milieu scolaire en mouvance. Il nous incombe de trouver les moyens pour les former à la flexibilité nécessaire à l'enseignement dans un tel milieu.

\section{Références}

Baker, C. (2011). Foundations of bilingual education and bilingualism (5 éd.). Clevedon : Multilingual Matters.

Boudreau, A., Dubois, L. et d'Entrebont, V. (2008). Représentation, sécurité/insécurité linguistique et éducation en milieu minoritaire. Dans P. Dalley et S. Roy (dir.), Francophonie, minorités et pédagogie (p. 145-175). Ottawa : Presses de l'Université d'Ottawa.

Boyer, H. (2006). Présentation. Études de linguistique appliquée, 3(143), 261-263. Repéré à https://www.cairn.info/revue-ela-2006-3-page-261.htm

Calvet, L.-J. (1999). Pour une écologie des langues du monde. Paris : Plon.

Francard, M., Geron, G. et Wilmet, R. (1993). L'insécurité linguistique dans les communautés francophones périphériques. Cabiers de l'Institut linguistique de Louvain, 19(3-4).

Mujawamariya, D. (2002). Les minorités visibles et ethnoculturelles sur la profession enseignante : un parcours parsemé d'embûches. Dans D. Mujawamariya (dir.), L'intégration des minorités visibles et ethnoculturelles dans la profession enseignante : récits d'expériences, enjeux et perspectives (p. 203-235). Québec, QC : Les Éditions Logiques. 